\title{
Sphex funerarius GUSSAKOVSKIJ nouveau pour la Belgique (Hymenoptera, Sphecidae)
}

\author{
Par Yvan Barbier * et Jelle Devalez **
}

\section{Introduction}

Sphex funerarius GussakovskiJ $(=S$. rufocinctus BRULLÉ 1883) est un sphécide largement répandu. Il est présent en Europe, en Afrique du Nord, en Asie occidentale et centrale, en Chine (Bitsch et al., 1997). En Europe, il est surtout représenté dans la région méditerranéenne mais se rencontre aussi en Europe centrale et, vers le nord, jusqu'au Danemark où une petite population relique semble subsister (Lomholdt, 1984).

Les femelles creusent leur nid dans le sol sablonneux. Celui-ci est composé d'une galerie oblique dans laquelle s'ouvrent une ou plusieurs cellules. Les proies sont des Tettigoniidae ou des Acrididae (Bitsch et al., 1997).

En France, ce Sphex est essentiellement présent dans le sud et le sud-ouest (Barbier, 2007). Il est connu depuis longtemps de la côte ouest qu'il remonte jusque dans le Calvados (Estuaire de l'Orne, A. Livory comm. pers.). Il est également bien présent dans les dunes de la Manche (obs. pers. en 1994). Dans l'est, des observations plus récentes nous montrent qu'il remonte à présent jusque dans le Bas-Rhin (Haguenau, août 2007, G. Coppa, comm. pers.) et le Haut-Rhin (Niederhergheim et Geisswasser, juillet 2007, M. Aubert, comme. pers) alors qu'il n'y était pas connu avant.

En Allemagne, alors que sa présence n'était plus établie depuis le début des années '60, il a été trouvé dans le sud du Baden-Wurtemberg dès le milieu des années '90 où il est bien implanté dans les dunes intérieures (Schmid-Egger, 1996; Freundt, 2002). En 2001, il a été découvert bien plus au nord, à Wesel en Rhénanie du Nord-Westphalie, également dans un biotope sablonneux et chaud (Freundt, 2002). L'espèce y est prédatrice de l'orthoptère Phaneroptera falcata. On le trouve aussi dans la Hesse en 2000 (Dressler, 2000), en Rhénanie-Palatinat en 2003 (Reder, 2003). Sphex funerarius semble être en expansion en Allemagne et utiliserait, à l'instar de Sceliphron curvatum, la vallée du Rhin comme voie de progression vers le nord-ouest (Schmid-Egger, 2005). Cette expansion est confirmée par Stolle et al. (2004) qui le citent du land de SaxeAnhalt alors qu'il n'y avait plus été observé depuis 1960.

\footnotetext{
* Faculté universitaire des Sciences agronomiques de Gembloux. Unité de gestion des resources forestières et des milieux naturels. Passage des déportés, 2. B-5030 Gembloux.

E-mail: yvan.barbier@gmail.com

** Labo voor Zoöfysiologie, Krijgslaan 281 S33 B-9000 Gent,

Belgique. E-mail: jelle.devalez@gmail.com
}

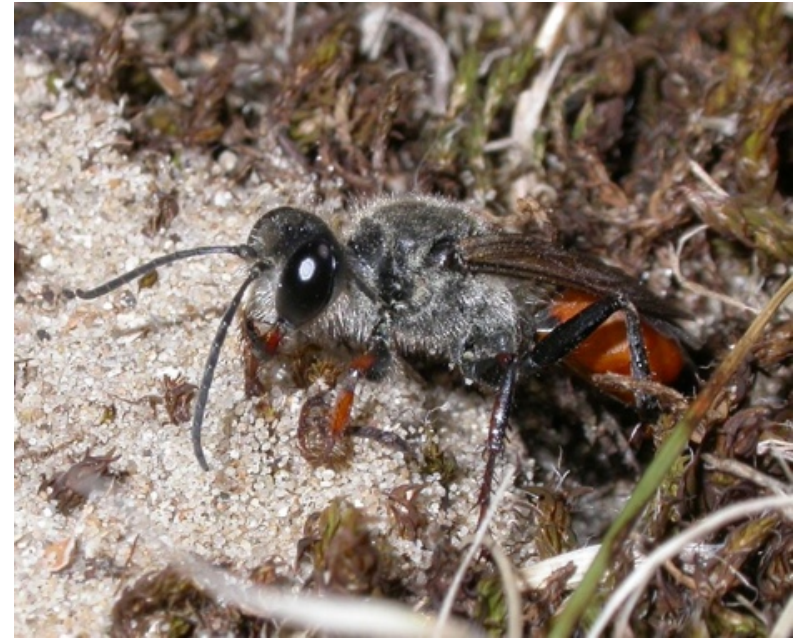

Figure 1. Femelle de Sphex funerarius à l'entrée de son nid (Photo J Devalez)

L'espèce est maintenant présente dans 9 landers (voir http://hymis.de).

Au Grand Duché de Luxembourg, il a été observé pour la première fois dans les environs de Düdelange, dans un biotope sablonneux et thermophile (Cungs \& Jakubzik, 2001). C'est la toute première mention pour le Benelux.

Ailleurs en Europe, il a été récemment signalé de Tchéquie par Straka et al (2004) alors qu'il n'y avait plus été observé depuis 40 ans. Il a également été trouvé pour la première fois en Lithuanie par Budrys (2001) et pour la première fois en Styrie (Autriche; Gusenleitner, 2000).

\section{Découverte en Belgique}

C'est dans ce contexte d'expansion que l'espèce a été observée pour la première fois en Belgique pendant l'été 2007 par l'un de nous (JD) entre le 20 juillet et le 3 août 2007. Bien que les effectifs y soient faibles, elle semble présente dans la plupart des complexes dunaires situés entre Koksijde (Coxyde) et la frontière néerlandaise (le Zwin). Elle a même été observée en train de nicher à Nieuwpoort (Nieuport) le 3 août 2007. A Oostduinkerke, elle a été observée à plusieurs reprises sur des inflorescences d'Eryngium maritimum.

Tout montre que l'espèce est bien implantée le long de la côte belge. Cette implantation est certainement récente car les observateurs ne manquent pas le long du littoral et jamais elle n'y avait été vue auparavant. 


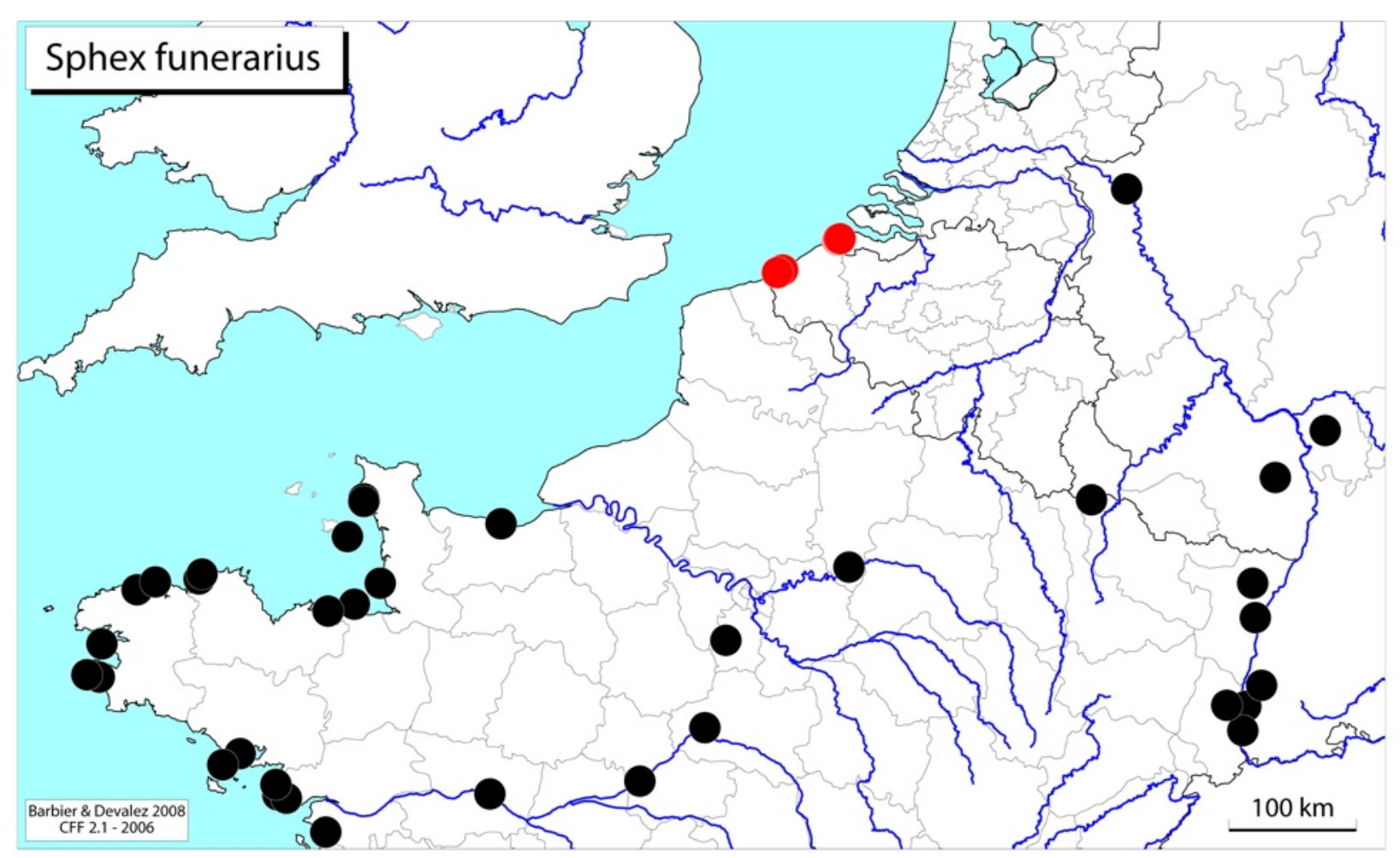

Figure 2. Carte de distribution des observations relatives à Sphex funerarius dans le nord de la France et les régions voisines. Les nouvelles données rapportées dans cet article sont indiquées en rouge.

Les observations récentes, faites à l'étranger (France, Luxembourg, Allemagne, Tchéquie, Lithuanie), semblent montrer qu'on assiste à une expansion de l'espèce vers le nord. On la redécouvre dans des régions où elle était considérée comme disparue mais aussi dans des régions où elle y était inconnue auparavant (est de la France par exemple). La population découverte à la côte belge est probablement issue d'individus qui seraient remontés le long de la côte française. L'espèce est donc à rechercher dans le Nord-Pas-de-Calais où on n'a pas encore d'observation. De même, il est fort probable que sa progression continue vers le nord et c'est donc aux Pays-Bas qu'il faudrait la rechercher.

Il est difficile de donner une explication à ce phénomène d'expansion mais il est probable qu'il ait un lien avec le réchauffement climatique. D'autres espèces méridionales connaissent, elles aussi, une expansion de leur aire de distribution vers le nord (par exemple Polistes dominulus; Barbier et al., 1995; Barbier \& Baugnée, 2002).

\section{Références bibliographiques}

Barbier Y, 2007. Atlas Hymenoptera, page "Sphex". http:// www.atlashymenoptera.net.

Barbier Y, Baugnée J-Y \& Rasmont P, 1995. La dérive faunique de Polistes dominulus (CHRIST) et de P. biglumis (L.) en Belgique et dans les régions limitrophes (Hymenoptera Vespidae). Notes Fauniques de Gembloux 30: 59-65.

Barbier Y \& Baugnée J-Y, 2002. Nouvelle estimation de l'expansion de Polistes dominulus (CHRIST) en Wallonie et régions limitrophes (Hymenoptera, Vespidae). Bulletin de l'Institut royal des Sciences naturelles de Belgique, 72-Suppl. : 187-188.

Bitsch J, Barbier Y, Gayubo SF, Schmidt K \& Ohl M, 1997. Faune de France. Hyménoptères Sphecidae d'Europe Occidentale, volume II. Fédération Française des Sociétés de Sciences Naturelles, Paris, 429pp, 76 planches, 154 cartes.
Budrys E, 2001. Five new for Lithuanian fauna species of Sphecidae and Crabronidae (Hymenoptera, Apoidea) - Pienkios naujos Lietuvos faunai žiedvapsvių (Hymenoptera, Apoidea: Sphecidae, Crabronidae) rūšys. Acta Zoologica Lituanica 11: 388-390.

Cungs J \& Jakubzik A, 2001. Seltene Weg- und Grabwespen (Hymenoptera, Aculeata: Pompilidae \& Sphecidae) Luxemburgs, mit besonderem Bezug auf das ehemalige Erzabbaugebiet Haardt bei Düdelingen. Bull. Soc. Nat. luxemb. 101: 117-128.

Dressler A, 2000. Grabwespen, Wegwespen und solitäre Faltenwespen (Hym.: Sphecidae, Pompilidae, Eumeninae) der Gemarkung Darmstadt-Eberstdt und angrenzenden Gebiete. Hessische Faunistische Briefe 19: 28-38.

Freundt R, 2002. Kommentierte Fundmeldung von Sphex funerarius GussaKovsKIJ, 1943 (Hymenoptera: Sphecidae), ehemals Sphex rufocinctus BRULLÉ, 1832. Neufund für NRW. Bembix 15: 19-21.

Guselneitner J, 2000. Hymenopterologische Notizen aus Österreich - 13 (Insecta: Hymenoptera aculeata). Linzer biol. Beitr. 32(2): 953-962.

Lomholdt O, 1984. The Sphecidae (Hymenoptera) of Fennoscandia and Denmark. Fauna Entomol. Scand. 4: 452pp.

Reder G, 2003. Seltene Hymenopteren bei Eisenbereg in der Nordpfalz, mit dem Wiederfund von Gorytes quadrifasciatus (FABR.) (Hymenoptera: Sphecidae, Apidae). Pollichia Kurier 19: 25-27

Schmid-Egger G, 1996. Neue oder bemerkenswerte südwestdeutsche Stechimmen-funde. Bembix 7: 18-20.

Schmid-Egger G 2005. Sceliphron curvatum (F. SMITH 1870) in Europa mit einem Bestimmungsschlüssel für die europäischen und mediterranen Sceliphron-Arten (Hymenoptera, Sphecidae). Bembix 19: 7-28.

Stolle E, Burger F \& Drewes B, 2004. Rote Liste der Grabwespen (Hymenoptera: "Sphecidae") des Landes Sachsen-Anhalt. Berichte des Landesamtes für Umweltschutz Schsen-Anhalt 39: 369-375.

Straka J, Bogush P, Tyrner $P$ \& Vepřek D, 2004. New important faunistic records of Hymenoptera (Chrysidoidea, Apoidea, Vespoidea) from the Czech Republic. Klapalekiana 143-153.

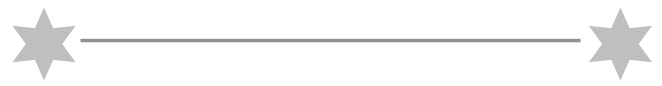

